\title{
Crystallinity reduction and enhancement in the chemical reactivity of cellulose by non-dissolving pre-treatment with tetrabutylphosphonium acetate
}

\author{
Daniel Rico del Cerro - Tetyana V. Koso - Tia Kakko • Alistair W. T. King (D) \\ Ilkka Kilpeläinen
}

Received: 21 August 2019/Accepted: 5 February 2020/Published online: 18 February 2020

(C) The Author(s) 2020

\begin{abstract}
Herein, we demonstrate the activation of commercial chemical cellulose pulps towards chemical modification by a pre-treatment step with tetrabutylphosphonium acetate $\left(\left[\mathrm{P}_{4444}\right][\mathrm{OAc}]\right)$. A heterogeneous (non-dissolving) pre-treatment was applied allowing for a significant reduction in crystallinity, without concomitant formation of the thermodynamically stable cellulose II. An increase in chemical reactivity was demonstrated using two model reactions; (1) acetylation (organic swelling conditions), where high degrees of substitution (DS) were obtained without the need for a catalyst, and (2) 4-acetamido-TEMPO oxidation (aqueous swelling conditions), where significant degrees of oxidation (DO) were obtained, beyond those for the untreated pulps. In both tests a notable improvement in cellulose reactivity was observed. Regioselectivity of acetylation was assessed using 2D NMR for one low and one
\end{abstract}

Electronic supplementary material The online version of this article (https://doi.org/10.1007/s10570-020-03044-6) contains supplementary material, which is available to authorized users.

D. R. del Cerro · T. V. Koso - T. Kakko ·

A. W. T. King $(\bowtie) \cdot$ I. Kilpeläinen $(\bowtie)$

Chemistry Department, University of Helsinki, AI

Virtasen Aukio 1 (Chemicum), 00560 Helsinki, Finland

e-mail: alistair.king@helsinki.fi

I. Kilpeläinen

e-mail: ilkka.kilpelainen@helsinki.fi high DS sample. The low DS showed a small degree of acetylation of the 6-OH, whereas, the high DS from the pre-treated sample showed mainly mixtures of triacetate and diacetates. Important mechanistic information is attained for future development of aqueous and organic-based reactions involving this ionic liquid pre-treatment.

Keywords Mercerization - Cellulose acetate . TEMPO oxidation · Ionic liquid $\cdot$ Pulp reactivity

\section{Introduction}

Cellulose is the most abundant natural polymer produced by living organisms and it is the most important skeletal component in plants, due to its rigid structure (Klemm et al. 2005). As the main component of biomass, cellulose has a major role in the nature's circulation of carbon, with plants acting as a huge sink for excess atmospheric $\mathrm{CO}_{2}$ (Pan et al. 2011). Thus, cellulose is considered to be a vast source of environmentally friendly, sustainable and biocompatible raw material (Klemm et al. 1998). The rigid highlyordered structure of crystalline cellulose provides desirable mechanical properties. However, the lack of a true melting point makes its low-cost processing very difficult. 
One way around this problem is to apply one of the well-known heterogeneous chemical modification methods for plasticization of cellulose. Cellulose nitrate formation, from reaction with nitric acid, is the classical example. However, these methods add cost to the final products. The accessibility and chemical reactivity of cellulose, under heterogeneous conditions, depend on several factors, such as the chemical composition, molecular weight distribution, crystallinity, porosity and morphology (fibrillar complexity) of the fibers (Krässig 1993).

Alternatively, cellulose can be dissolved in a suitable direct dissolution solvent. However, the complexity of cellulose solvents is such that they are typically expensive, toxic, and often not tolerant towards many chemical reagents or are simply difficult to recycle. $N$-methylmorpholine- $N$-oxide (NMMO) is a well-known low-cost and low-toxicity bulk solvent for cellulose, already used for many years in an industrial cellulosic fibre dissolution/regeneration process (Lyocell process) (Rosenau et al. 2001). There are also a limited number of electrolytic or reactive solvent systems, such as DMSO/TBAF (Ramos et al. 2005), DMAc/LiCl (Araki et al. 2006), $\mathrm{DMF} / \mathrm{N}_{2} \mathrm{O}_{4}$ (Philipp et al. 1987) and some inorganic molten salt hydrates, such as $\mathrm{LiClO}_{4} \cdot \mathrm{H}_{2} \mathrm{O}$ (Fischer et al. 2003), capable of dissolving cellulose. All of these have quite hazardous components.

However, in the recent decades, ionic liquids (ILs) and deep eutectic solvents (DESs) have been considered as new solvents for cellulose dissolution and biomass processing. DESs are often cheaper than ILs. Those based on choline chloride, at least, are also low toxicity. However, they suffer a few major drawbacks in biomass processing; Low toxicity choline-based structures are rather thermally unstable and the solubility of cellulose in DESs is very poor, compared with the solubility in key ILs (De Oliveira Vigier et al. 2015; Chen et al. 2019). Nevertheless, DESs still have potential in the biomass research field (Chen and $\mathrm{Mu}$ 2019). Initial investigations have demonstrated applications that do not require cellulose dissolution capabilities, in particular those that allow for enhanced fibrillation ( $\mathrm{Li}$ et al. 2017) or chemical modification (Li et al. 2018) of lignocellulosics.

ILs have already been well studied as novel solvents for cellulose dissolution and biomass processing (Swatloski et al. 2002; Lasse et al. 2013; Holding et al. 2014, 2016, 2017). ILs are often defined as 'molten organic salts' with melting points below $100{ }^{\circ} \mathrm{C}$ (Skoda-Földes 2014; Chowdhury et al. 2007; Siódmiak et al. 2012), whose chemical and physical properties can be tuned by varying the cations, homologous substituents and anions. Having essentially no vapor pressure, they are non-flammable and in contrast to traditional volatile organic compounds, they are often spuriously described as 'green' solvents (Zhu et al. 2006). However, they are known to dissolve a wide range of organic compounds; from small polar or non-polar molecules to rigid amphiphilic polymers, such as cellulose.

The ability of ILs to dissolve cellulose is clearly dependent on the high basicities imparted by the anions (Fukaya et al. 2006, 2008; King et al. 2012; Parviainen et al. 2013). However, there are some basic ILs which require a dipolar aprotic co-solvent to facilitate dissolution (Holding et al. 2014, 2016, 2017). Of these phosphonium homologues, $\left[\mathrm{P}_{4444}\right][\mathrm{OAc}]$ (Fig. 1) is among the lowest toxicity of the series towards several cell lines (Ruokonen et al. 2016; Witos et al. 2017) and comparable to the toxicity of various imidazoliumbased structures. Tetraalkylphosphoniums also have higher thermal and chemical stabilities than the commonly used imidazolium series. [bmim][OAc], as a common imidazolium analogue for cellulose dissolution, is known to react with cellulose via imidazolium-C2 reactivity (Ebner et al. 2008; Rico del Cerro et al. 2018; Clough et al. 2015). Therefore, there are important advantages to phosphonium-based ILs, if they can find application in cellulose processing.

Previously, the reduction in crystallinity after the non-dissolving pre-treatment of microcrystalline cellulose (MCC) with tetrabutylphosphonium acetate $\left(\left[\mathrm{P}_{4444}\right][\mathrm{OAc}]\right.$, Fig. 1) has been shown (Holding et al. 2016; Pena et al. 2019). This led to increased enzymatic digestibility using cellulases.

Herein, we present the increased chemical reactivity of high molecular weight technical kraft pulps, towards acylation (organic swelling conditions) and nitroxyl radical-catalysed oxidation (aqueous conditions), after their pre-treatment with pure $\left[\mathrm{P}_{4444}\right][\mathrm{OAc}]$ (non-dissolving conditions). The effect of the pretreatment on the regioselectivity of acetylation is shown and on the morphology of the fibre structure during aqueous oxidation. The results show clear potential for increased reactivity under heterogeneous reaction conditions. 


\section{Experimental}

Materials and methods

Three cellulosic pulps were used in our reactivity studies; Enocell (bleached hardwood pre-hydrolysis kraft pulp, Enocell Mill, Uimaharju, Stora Enso, 6.8\% xylan), birch (bleached birch kraft pulp, UPM Kaukas Mill, $87.7 \% \alpha$-cellulose) and eucalyptus (eucalyptus kraft pulp, UPM Fray Bentos Mill, 87.4\% $\alpha$-cellulose). $\left[\mathrm{P}_{4444}\right][\mathrm{OAc}]$ was prepared according to the literature procedure (King et al. 2018). All the reagents and solvents were high purity ( $\geq 98 \%$ ) and were used as obtained from the commercial suppliers, without further purification. Selected AcNH-TEMPO-oxidized cellulosic samples were studied with the help of Olympus BX51TF microscope, equipped with DP70 colour camera and adjustable polarized lenses. Cross-polarizer angle was optimized for better image contrast, producing differences in colour.

Products were characterized by Attenuated Total Reflection Infra-Red spectroscopy (ATR-IR) and liquid-state NMR spectroscopy on a Bruker NEO Avance (600 MHz ${ }^{1} \mathrm{H}$-frequency), using either DMSO-d $_{6}$ or $\left[\mathrm{P}_{4444}\right][\mathrm{OAc}]: \mathrm{DMSO}_{6} \mathrm{~d}_{6}(20: 80 \mathrm{wt} \%)$ (King et al. 2018), to dissolve those samples that were not soluble in DMSO- $\mathrm{d}_{6}$ alone (typically low DS samples). Wide-angle X-ray scattering (WAXS) measurements were performed on a PANalytical X'Pert Pro MPD system. The diffracted intensity of $\mathrm{Cu} \mathrm{K} \alpha$ radiation ( $\lambda=1.54 \AA$, under a condition of $45 \mathrm{kV}$ and $40 \mathrm{~mA}$ ) was measured in a $2 \theta$ range between $5^{\circ}$ and $50^{\circ}$. Molar mass determination was performed by GPC with an Agilent Infinity 1260, using LiCl/DMA as mobile phase, according to previous literature (Kakko et al. 2017; Kakuchi et al. 2015, 2017). (See Sect. 7 of SI).

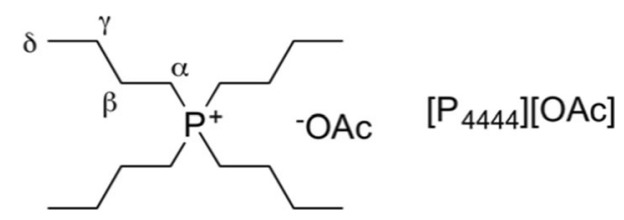

Fig. 1 Structure and position assignment for the ionic liquid tetrabutylphosphonium acetate $\left(\left[\mathrm{P}_{4444}\right][\mathrm{OAc}]\right)$, used in this study for crystallinity reducing pre-treatments and liquid state NMR analyses
IL pre-treatment of cellulose pulps

A glass pressure tube was charged with the desired amount of $\left[\mathrm{P}_{4444}\right][\mathrm{OAc}]$, and melted at around $80{ }^{\circ} \mathrm{C}$. Once the IL was melted, $10 \mathrm{wt} \%$ of the desired pulp was soaked into the IL. The mixture was homogenized using a rotary homogenizer and heated at $120{ }^{\circ} \mathrm{C}$ for $5 \mathrm{~h}$. The pre-treated pulp was stored in a sealed flask under argon atmosphere for further homogeneous experiments. Additionally, a portion of this material was thoroughly washed with distilled water to remove the IL, filtered, dried under vacuum and stored in a sealed flask prior to further heterogeneous reactions.

Crystallinity index determination

The crystallinity index (CI) is frequently reported using the Segal method (Segal et al. 1959) as a simple data processing method to measure crystallinity values. However, its accuracy still open to discussion (Nam et al. 2016), which has motivated the use of other methods, such as those based on deconvolution (with or without amorphous subtraction) of the $1 \mathrm{D}$ data, or ${ }^{13} \mathrm{C}$ CP-MAS NMR spectra with $\mathrm{C} 4$ signal deconvolution (Park et al. 2010).

In our studies the $\mathrm{CI}$ is calculated by deconvolution (pseudoVoigt fitting) of the 1D X-ray diffractograms, over $2 \theta$ values of $5^{\circ}-50^{\circ}$. A suitable amorphous standard is critical, as is the glass background support. Therefore, we prepared an amorphous standard, using the higher purity pre-hydrolysis kraft pulp (Enocell), by dissolution and regeneration of the pulp in/from trifluoroacetic acid (TFA) (See Sect. 4a of SI). This method also requires a curve fitting software to separate the background, amorphous and crystalline contributions to the diffractograms. We used 'Fityk' (Wojdyr 2010), capable of peak and curve-fitting of 1D data. The flexibility of the software is such that it allows us to define single functions, for use within Fityk fitting, representing both the variable background and synthetic amorphous functions. This is achieved by defining an equation for a linear combination of functions (LCOF), required to fit the background and amorphous diffractograms (SI Sect. 4b). When fitting these LCOFs to the actual experimental data, the LCOF can be represented by a single variable (in our case height, corresponding to a specific total area of the LCOF), which can then be used in calculation of the CI values, during the final 
deconvolution-fitting procedure. Thus, we are able to fit the crystalline phases, glass background and a predefined 'amorphous' phase. Commonly used functions for deconvolution of the diffraction pattern are Gaussian, Lorentzian and Voigt (pseudoVoigt for faster data processing). Functionals are typically applied to the peak areas that contain the five diffraction planes corresponding to the (11̄0), (110), (012), (200) and (004) Miller indices (French 2014). (See Sect. 4b of SI). In our case we fitted these with pseudoVoigt functions (30:70 Gaussian:Lorentzian) and 2 extra peaks which represent crystallinity contributions in the regions from $35^{\circ}$ to $50^{\circ}$. These were fitted alongside the glass background and experimental amorphous contributions. The full procedure is given in the SI.

CI values are calculated according to Eq. (1), as the $\%$ ratio of the area of the crystalline signals $\left(A_{\mathrm{Cel}-\mathrm{I}}\right)$ to the total area $\left(A_{T o t}\right)$.

$C I=100 \times\left(A_{C e l-I} / A_{T o t}\right)$

\section{Acetylation}

Reactivity studies of pre-treated and non-treated cellulose pulps were performed by reacting $\sim 1 \mathrm{wt} \%$ of pulp at $165{ }^{\circ} \mathrm{C}$ for $18 \mathrm{~h}$ in either of two different acetylating agents, acetic anhydride (AA) and the less reactive isopropenyl acetate (IPA). This high dilution procedure was designed to assess changes in reactivity of the pulps and is not intended to be a preparatory procedure. Three different approaches for each type of pulp were conducted in parallel. These include reaction of (1) the untreated pulp (heterogeneous reaction), (2) the pre-treated pulp, with the consecutive aqueous IL removal step (heterogeneous reaction, IL-PT-W) and (3) the pre-treated pulp without the consecutive IL removal step (IL-PT-NW). This is termed as a 'homogeneous reaction' by the virtue of the fact that when acetylating agent is added, the cellulose rapidly swells and mostly dissolves during the reaction. The full procedures are given in Sect. $5 \mathrm{~b}$ $\& 5 \mathrm{c}$ of SI.

The acetylation products were analyzed by ${ }^{1} \mathrm{H}$ NMR for quantification of DS values, and by HSQC (Heteronuclear Single-Quantum Correlation) and HSQC-TOCSY (Total Correlation Spectroscopy) for regioselectivity determination (See Sect. $5 \mathrm{~d}$ of SI).
The degree of substitution (DS) of the samples were obtained by integration of the ${ }^{1} \mathrm{H}$ cellulose backbone spectral region $\left(I_{C}, 5.82-2.80 \mathrm{ppm}\right)$ and the acetate spectral region $\left(I_{A}, 2.40-1.60 \mathrm{ppm}\right)$. The DS values were calculated according to Eq. (2).

$D S=\left(I_{A} / 3 / I_{C} / 7\right)$

For those samples that were soluble in DMSO- $\mathrm{d}_{6}$ (DS range 1.61-2.92), Eq. 2 was applied directly. For the samples that were not soluble in DMSO- $\mathrm{d}_{6}$ (DS range $0-0.42$ ) the electrolyte $\left[\mathrm{P}_{4444}\right][\mathrm{OAc}]: D M S O-\mathrm{d}_{6}$ (20:40 wt\%) (King et al. 2018), was used to dissolve the samples and the integral values were obtained after running the diffusion-edited ${ }^{1} \mathrm{H}$ spectra. As the diffusion-edited ${ }^{1} \mathrm{H}$ has the tendency to overestimate integrals for faster moving species (acetate Me vs cellulose backbone $\mathrm{CHs}$ ), a correction factor was applied to these results. This was determined by linear correlation of the DS values, determined through the diffusion-edited experiments (in electrolyte) vs standard ${ }^{1} \mathrm{H}$ experiment (in DMSO- $\mathrm{d}_{6}$ ). Three reference samples of known DS $(0.7,1.30,1.92)$ were synthesized from the Enocell pulp (94\% cellulose), for this purpose. (See Sect. 5a of SI).

\section{AcNH-TEMPO-oxidation}

Oxidation of the raw and pre-treated materials was achieved using the AcNH-TEMPO/ $\mathrm{NaClO} / \mathrm{NaClO}_{2}$ system at $\mathrm{pH}$ 5.8, as described in previous literature (Kitaoka et al. 1999; Saito et al. 2010; Hirota et al. 2009). Acidic oxidation conditions, in the presence of $\mathrm{NaClO}_{2}$, was applied, to allow for conversion of intermediated aldehydes/acetals to carboxylates. A further acidification protocol was applied to all the samples in preparation for GPC analyses, as polymeric sodium salts typically do not dissolve in LiCl/DMA. Full procedures are given in Sect. 6 of SI.

\section{Degrees of oxidation}

The degree of oxidation $\left(\%_{\mathrm{DO}}\right)$ of individual fractions, represented as the $\%$ of the theoretical maximum and the values in $\mathrm{mmol} / \mathrm{g}$, derived from the $\mathrm{AcNH}-$ TEMPO oxidations, were determined from deconvolution of the ATR-IR data (carbonyl vs $\mathrm{C}-\mathrm{O}$ stretch regions). For individual peak area determinations, we used 'Fityk' (Wojdyr 2010). The data is regarded as 
semi-quantitative as a correlation between the IR data and conductometric titration (directly yielding the carboxylate content in $\mathrm{mmol} / \mathrm{g}$ ) was performed for key samples. Detailed data processing is provided in the Sect. 6 of SI.

\section{Results and discussion}

Three model kraft pulps were used in this study: (1) Enocell bleached hardwood (birch and $\sim 4 \%$ aspen) pre-hydrolysis kraft pulp (B-H-PHK-P, 6.8\% xylan), (2) Birch bleached hardwood kraft pulp (B-H-K-P, $20.8 \%$ xylan), Eucalpytus bleached hardwood kraft pulp (B-H-K-P, 26.2\% xylan). As with our previous studies on tetraalkylphosphonium acetate ionic liquids (Holding et al. 2014, 2016, 2017, 2018; Pena et al. 2019), we observed that the pre-treatment of three different kraft pulps with pure $\left[\mathrm{P}_{4444}\right][\mathrm{OAc}]$ at $120{ }^{\circ} \mathrm{C}$ did not dissolve the material, but rather the material became partially swollen during the IL pre-treatment. After washing away the IL from the samples, the dried materials still retained similar appearance as the untreated samples. In Pena et al. (2019) the authors pressed the sample free of the bulk of the IL. This feature is important as it reduces the amount of washing water required in the recovery of the pretreated cellulose. This would have a benefit in recycling of the system, reducing energy costs for water evaporation. However, in the present study we either directly washed away the IL, without pressing, to simply yield IL-free material for reactivity assessment or we retained the IL in the matrix during reaction. This latter sample series was to observe the effect of water washing/swelling and drying on the reactivity as it is known that hornification can occur upon drying of pulps, which significantly impedes their reactivity.

\section{Crystallinity reduction through IL pre-treatment}

The removal of crystallinity, under the non-dissolving conditions, was achieved by soaking $10 \mathrm{wt} \%$ of the different cellulose samples in pure $\left[\mathrm{P}_{4444}\right][\mathrm{OAc}]$, at $120{ }^{\circ} \mathrm{C}$ for $5 \mathrm{~h}$ (no agitation). Samples for crystallinity analysis were thoroughly washed with water and freeze-dried. For comparison, under dissolving conditions, $5 \mathrm{wt} \%$ of the pulps were suspended in $\left[\mathrm{P}_{4444}\right][\mathrm{OAc}]: D M S O-\mathrm{d}_{6} \quad(35: 65 \mathrm{wt} \%)$, at $65^{\circ} \mathrm{C}$ overnight (with magnetic stirring). After dissolution, the samples were regenerated into water using an Ultraturrax high-shear mixer. The high-shear was used to ensure rapid regeneration the cellulose, avoiding cellulose II crystallization. The samples were then subjected to 1D wide-angle x-ray scattering (WAXS, $5^{\circ}-50^{\circ}$ ) to allow for determination of crystallinity changes, by peak deconvolution.

Samples for WAXS were initially prepared by loading $50 \mathrm{mg}$ of freeze-dried samples into a $\mathrm{KBr}$ press (typically used for preparation of $\mathrm{KBr}$ discs for transmittance FT-IR analysis) and pressed for $45 \mathrm{~s}$ at 9 psi. The samples were placed on a glass stage and the height calibrated for surface reflection. The method used for crystallinity index (CI) determination was a sequence involving no baseline correction and fitting (optimization) of functions representing the glass background and amorphous contribution, alongside 7 pseudoVoigt functions, corresponding to the main diffraction planes in cellulose I (see the SI for the full procedure). TFA dissolution/regeneration was used to yield a sample as close to amorphous as possible (Sect. 3 of SI), for the amorphous background contribution. This diffractogram (Fig. S2 in the SI) still shows some periodic ordering (peak maximum at $20.6^{\circ}$ ) that cannot be attributed to actual extended crystalline regions and if a single Gaussian function (FWHM = 8) was fitted to the peak maximum, the periodic plane size would only be $\sim 11 \AA$, corresponding roughly to the length of one cellobiose unit. This is characteristic of 'amorphous' cellulose samples prepared through alternative methods, e.g. ballmilling or regeneration from trimethylsilylcellulose. It is a remnant of the crystallinity that cannot be removed with simple treatments. Herein, we consider this to be 'amorphous'. While this offers an ambiguous definition of cellulose crystallinity, through WAXS analysis, this is consistent with previous reports using amorphous standards. The X-ray diffraction patterns for the raw materials and IL pre-treated samples are shown in Fig. 2. The main diffraction peak regions for cellulose I $\left(1 \overline{1} 0\right.$ at $\sim 14.5^{\circ}, 110$ at $\sim 16.5^{\circ}, 012$ at $\sim 20.5^{\circ}, 200$ at $\sim 23.0^{\circ} \& 004$ at $\sim 34.5^{\circ}$ ) (French 2014) were used in the calculation, alongside two additional broad peaks in the 34-50 region, representing the crystalline contribution in these regions. 
The IL pre-treated Enocell pulp (Fig. 2a) exhibits mainly one broad asymmetric diffraction peak, very similar to the amorphous reference (Fig. S2 in the SI). However, small shoulders, corresponding to the (110) and (1 $\overline{1} 0)$ diffraction planes in cellulose $I$, are still present but are more obvious in the IL pre-treated birch and eucalyptus pulps (Fig. 2b, c, respectively). None of the samples show the characteristic diffraction planes for cellulose II, the most obvious of which would be the $(1 \overline{1} 0)$ plane at $\sim 12.5^{\circ}$. However, a slight splitting or shoulders on the (200) peak can be observed indicating some small amount of cellulose II phase formation. The results indicate that the crystallinity was most efficiently removed from the higher purity Enocell pulp after non-dissolving pre-treatment. In the case of the kraft pulps (not pre-hydrolysed), surface adsorbed hemicelluloses or increased molecular weight may be a restricting factor for decrystallisation. However, when the samples pretreated by dissolution and regeneration (Fig. 2d-f) were analyzed, there is clearly a larger reduction in scattered x-rays for these samples compared to the non-dissolving pre-treatment. This is concomitant with a large reduction in intensity corresponding to the main cellulose I diffraction planes, as with the nondissolved pre-treated samples. However, when the diffractograms are deconvoluted, the average (of 3 pulps) CI values are actually slightly higher than for the non-dissolving pre-treatments giving $30.4 \%$ and $27.6 \%$ crystallinity, respectively. After measuring the height of the pressed discs for the untreated and dissolved/regenerated samples, it is clear that the densities of the materials recovered after the pretreatments are different, allowing for differences in scattering intensity. The untreated samples had average (of 3 pulps) disc heights of $0.28 \mathrm{~mm}$ and the dissolved/regenerated disc height average was $0.49 \mathrm{~mm}$. This suggests that the dissolution/regeneration procedure produces a more porous material, which may be beneficial for chemical conversion.

In addition, the average crystallite sizes for the main (200) peak, calculated using the fitted peak fullwidth half maximum (FWHM) values and the Scherrer equation (see SI, Eq. 2), are smaller for the dissolved and regenerated samples $(2.4 \mathrm{~nm})$ than for the nondissolving pre-treatment $(3.7 \mathrm{~nm})$ or the untreated pulps $(4.2 \mathrm{~nm})$. Thus, while the crystallinity values for the dissolved and regenerated samples are slightly higher than for the non-dissolved samples, the crystallite sizes are smaller indicating more effective disruption of the crystalline structure, than is indicated by the CI values alone. This smaller crystallite size may be representative of the fact that these samples were homogenized (fully dissolved) and rapidly regenerated using high shear. Whereas, the nondissolving pre-treatments are more akin to a Mercerization-type process (heterogeneous in nature), where outer polymeric surfaces of the elemental crystallites/fibrils are etched/swollen first. In the non-dissolving IL case, the surface chains are likely slowly swollen into the bulk amorphous phase. This most likely consists of a mass transfer-restricted bulk of partially solvated cellulose chains in glassy IL, which do not have the mobility to reorient into antiparallel motifs, as required for conversion to cellulose II and what is observed for Mercerization. After washing away the IL, the samples then simply regenerate in place. Thus, the larger residual (200) crystallite sizes observed for the non-dissolved pre-treated samples, compared to the dissolved and regenerated samples, are likely the residual core crystallites that are not extracted into the bulk due to mass transfer limitations. The full crystallite sizes are given in the SI (Table S2).

Changes in crystallinity due to increase or decrease in the hemicellulose content were also ruled out. Xylan is the main hemicellulose in hardwood kraft pulps. We determined the xylan contents (wt\%) by quantitative ${ }^{1} \mathrm{H} \quad \mathrm{NMR}$ analysis in $\left[\mathrm{P}_{4444-}\right.$ ][OAc]:DMSO-d 6 (20:80 wt\%) (King et al. 2018). The ${ }^{1} \mathrm{H}$ data was spline baseline corrected and deconvoluted, by applying Gaussian functions for the polymeric anhydroglucose $\mathrm{H} 1$ and anhydroxylose $\mathrm{H} 1$ peaks. Assuming the xylan contained no other sugar units, this allowed for calculation of the wt $\%$ xylan contents for the untreated and pre-treated pulps (Table 1). The results show that there are only slight reductions in xylan contents after the pre-treatments. Full details are given in the supporting information.

The results from the crystallinity analysis are shown in Fig. 3. Naturally we must assume that there is error in this calculation due to the poor peak resolution that the small crystallite sizes afford (poor resolution of peaks) and the number of different phenomena that affect peak shape. However, it is clear that the crystallinities of the pre-treated Enocell, birch and eucalyptus pulps were reduced significantly. Next 


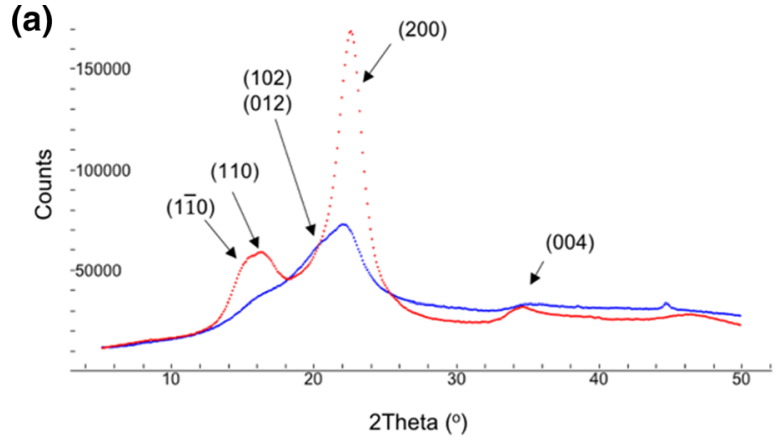

(c)

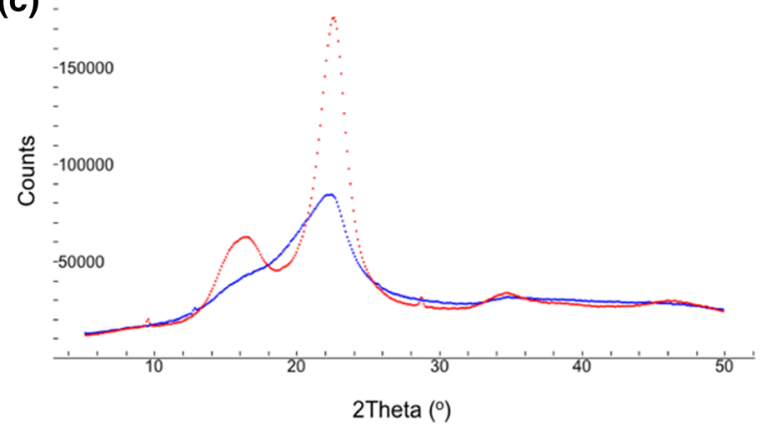

(e)

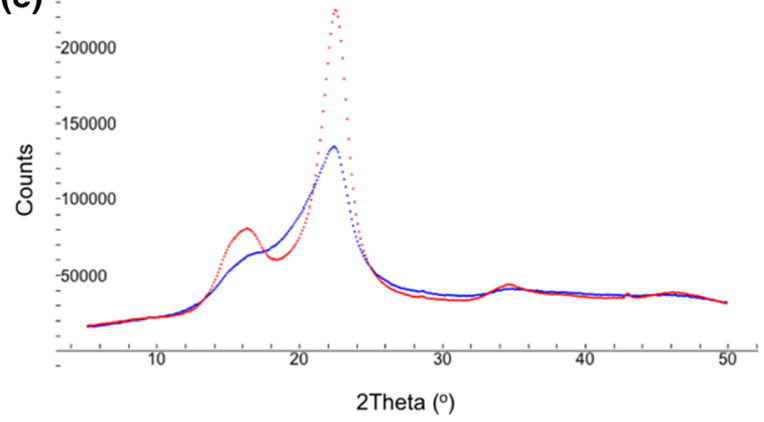

Fig. 2 WAXS diffractograms for untreated pulps (red) and pretreated pulps (blue): a Enocell B-H-PHK-P after non-dissolving pre-treatment. b birch B-H-K-P pulp after non-dissolving pretreatment, c eucalyptus B-H-K-P pulp after non-dissolving pre-

was to demonstrate if there is an enhancement in chemical reactivity.

Enhanced reactivity

To assess the reactivity of the pre-treated cellulose, two model reactions were chosen; (1) an acetylation reaction under organic swelling conditions and (2) nitroxyl radical-mediated oxidation (aqueous conditions). These reactions were chosen due to the importance and wide application of these cellulose derivatives (Fischer et al. 2008; Jelínková et al. 2002; (b)

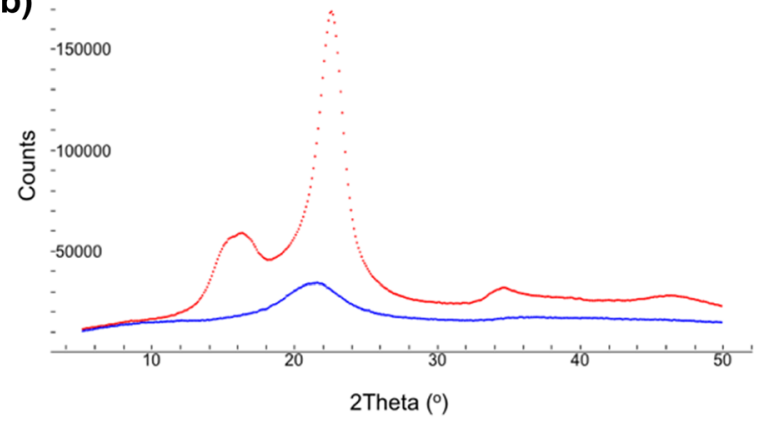

(d)

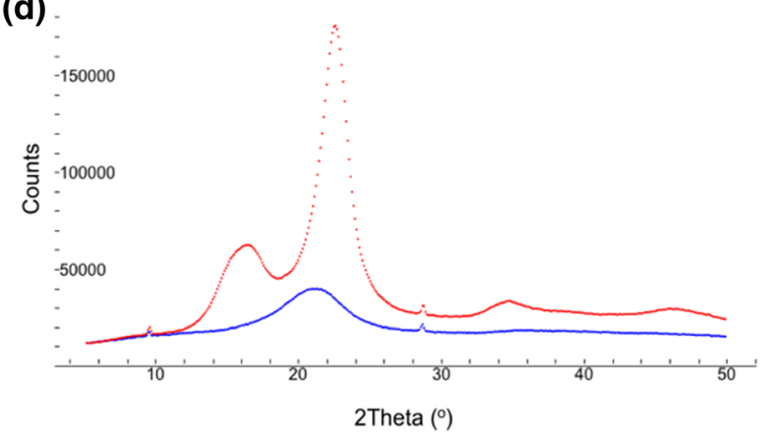

(f)

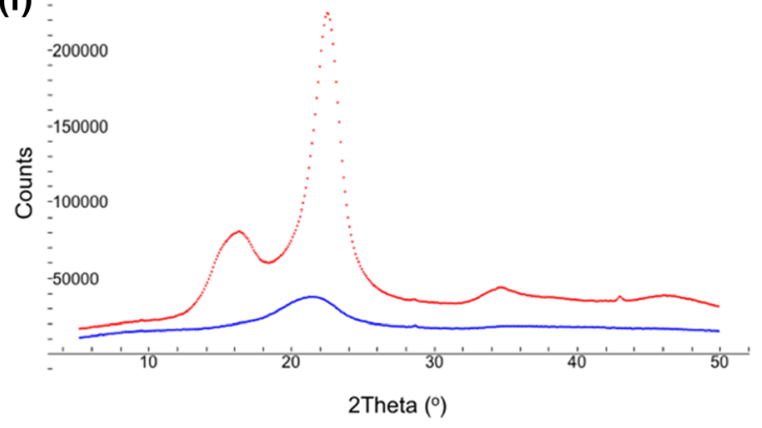

treatment, d Enocell B-H-PHK-P after dissolving pre-treatment, e birch B-H-K-P pulp after dissolving pre-treatment, f eucalyptus B-H-K-P pulp after dissolving pre-treatment

Zimnitsky et al. 2006). Acetylation of cellulose has been widely studied in common non-derivatising cellulose solvents (Heinze et al. 2003; Jebrane and Sèbe 2007; Jebrane et al. 2011; Chen et al. 2016). This reaction is commonly performed in the presence of catalysts (Jebrane et al. 2011; Li et al. 2009; Chen et al. 2014) with different acetylating agents, such as AA (Wu et al. 2004; Nawaz et al. 2014; Jogunola et al. 2016; Granström et al. 2008), and recently with vinyl acetate (VinAc) or IPA (Kakko et al. 2017; Kakuchi et al. 2015, 2017). The same reaction is commonly performed industrially using AA and catalytic 
sulphuric acid, under heterogeneous conditions. Selective oxidation of cellulose at C6 has been heavily studied using the popular 2,2,6,6-tetramethylpiperidine-1-oxyl (TEMPO) (Isogai and Kato 1998; Isogai et al. 2011; Orelma et al. 2012; Fukui et al. 2018; Guo et al. 2018) nitroxyl radical catalyst or the much more water soluble 4-acetamido-2,2,6,6tetramethylpiperidine-1-oxyl (AcNH-TEMPO) catalyst (Kitaoka et al. 1999; Saito et al. 2010; Hirota et al. 2009). The latter is what we used in our studies due to its higher activity.

\section{Acetylation}

The non-dissolving IL pre-treated and raw (untreated) pulps, as reference, were acetylated using both AA and IPA as acetylation reagents ( $1 \mathrm{wt} \%$ pulp consistency at $165{ }^{\circ} \mathrm{C}$ for $18 \mathrm{~h}$ ). Two different types of pre-treated samples were used: (1) a 'heterogeneous route' where the samples were IL pre-treated and washed free of IL (IL-PT-W), (2) a 'homogeneous route' where the samples were IL pre-treated but not washed (IL-PT$\mathrm{NW}$ ), to retain the swollen state of the molecules and observe the difference in reactivity that this allows. This is not technically a homogeneous reaction as the reaction starts off in the solid state and then progresses into solution over a period of time. These schemes led to DS values ranging from 0 to 2.99 .

Unfortunately, there is not one rapid method that allows for DS determination over such a wide range. Recently, a correlation of ATR-IR carbonyl vs C-O stretch peak height ratios and more accurate DS determination through ${ }^{31} \mathrm{P}$ NMR spectroscopy has been used for DS determination over a wide DS range (Kakko et al. 2017; Kakuchi et al. 2015, 2017, King et al. 2010). However, ATR-IR suffers from several sources of error, such as having only a fixed sample penetration depth next to the reflection surface, leading to errors with heterogeneous samples. In addition, there are no general calibration standards available for acetylated celluloses and significant error can be introduced to the data, based on the data processing method applied. Therefore, to further improve confidence in the present results, baseline correction and peak deconvolution was applied to the IR data. The ATR-IR results were also compared with DS values obtained from ${ }^{1} \mathrm{H}$ NMR integration over the whole DS range (Fig. 4). Further complications arise from the fact that we use relatively high purity cellulose (Enocell B-H-PHK-P, $\sim 6 \%$ hemicellulose) vs kraft pulps ( 20-25\% hemicellulose). Thus, a general DS scale, based on a maximum of 3 for cellulose triacetate, is not totally suitable but kept for simplicity to illustrate the trends in Fig. 4, as we are dealing with DS values ranging from very low to very high.

As some of the low DS value products, obtained mainly through the heterogeneous methods used in this article, were not soluble in DMSO- $\mathrm{d}_{6}$ it was necessary to dissolve these samples into the $\left[\mathrm{P}_{4444-}\right.$ ][OAc]:DMSO-d $\mathrm{d}_{6}(20: 80 \mathrm{wt} \%)$ electrolyte for NMR analysis (King et al. 2018). This allowed for integration of the acetate versus cellulose backbone region from the diffusion-edited ${ }^{1} \mathrm{H}$ spectrum (King et al. 2018). This required application of a correction factor which, although introduces further error, is necessary as the diffusion-editing tends to overestimate the degree of acetylation. This is due to large differences in relaxation between the polysaccharide backbone protons and the acetate methyl protons causing disproportionate signal losses, prior to acquisition (King et al. 2018). The combination of the ATR-IR deconvolution and NMR methods was applied to give more confidence to the general trends, in the form of

Table 1 wt $\%$ xylan contents, based on ${ }^{1} \mathrm{H}$ NMR analysis and quantification of the anhydroxylose unit (AXU) H1 peaks and the number-average molecular weights $\left(\mathrm{DP}_{\mathrm{N}}\right)$, from GPC analysis

\begin{tabular}{|c|c|c|c|c|c|c|}
\hline \multirow[t]{2}{*}{ Pulp } & \multicolumn{2}{|c|}{ Enocell B-H-PHK-P } & \multicolumn{2}{|l|}{ Birch B-H-K-P } & \multicolumn{2}{|c|}{ Eucalyptus B-H-K-P } \\
\hline & Untreated pulp & IL PT $W^{\mathrm{a}}$ & Untreated pulp & IL PT $\mathrm{W}^{\mathrm{a}}$ & Untreated pulp & IL PT $W^{\mathrm{a}}$ \\
\hline Xylan content (wt\%) & 6.8 & 5.4 & 26.2 & 22.8 & 20.8 & 18.9 \\
\hline $\mathrm{DP}_{\mathrm{N}}(\mathrm{g} / \mathrm{mol})$ & 330.4 & 272.5 & 330.9 & 320.3 & 365.7 & 273.5 \\
\hline
\end{tabular}

${ }^{a}$ IL-PT-W is the IL pre-treated pulp with a further washing step to remove the IL before the addition of the acetylating reagent (heterogeneous route) 


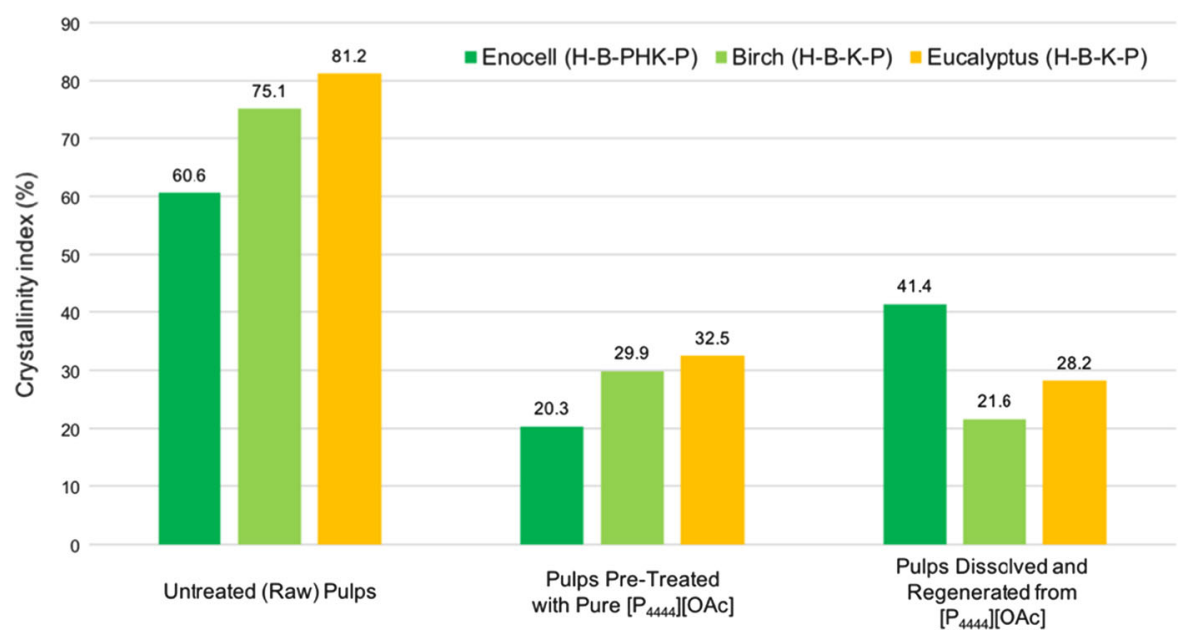

Fig. 3 CI values of the untreated and pre-treated pulps

2D plots (IR data vs NMR data, Fig. 4). For ease of reading, the data points are color coded in three separate graphs, representing the pre-treatment type, pulp source and acetylation reagent. The data is also tabulated for numerical reference (Table 2). The DS values from the NMR integration methods show a good linear correlation with the ratios from the deconvolution of the ATR-IR absorbance.

From the washed pre-treated samples (IL-PT-W), the Enocell and birch, reacted with AA, clearly show increased reactivity over the untreated samples. The DS values were shown to be 1.61 and 1.90 for pretreated birch and Enocell, but 0.18 and 0.32 for untreated birch and Enocell pulps, respectively. ILPT-W eucalyptus acetylated with AA only gave a DS value of 0.19 . The IPA reactions did not show significant enhancement in reactivity for the IL-PT$\mathrm{W}$ samples. This shows that increase in reactivity is possible with the IL pre-treatment, specifically with the more reactive reagent, AA, where some autocatalysis may occur.

The 'homogeneous route', where the cellulose samples were dissolved (IL-PT-NW) clearly shows higher reactivity than the heterogeneous route (IL-PT$\mathrm{W})$, see Table 2 . The differences in reactivity between the homogeneous route (IL-PT-NW) and heterogeneous route (IL-PT-W) are not unexpected, as cellulose in the heavily swollen state should give much easier access to all hydroxyls, whereas, typical heterogeneous acetylation reactions, in the absence (a)

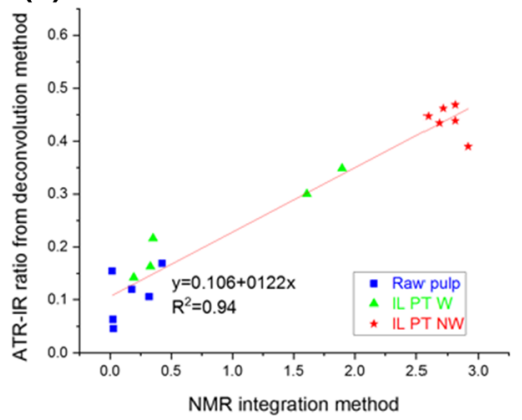

(b)

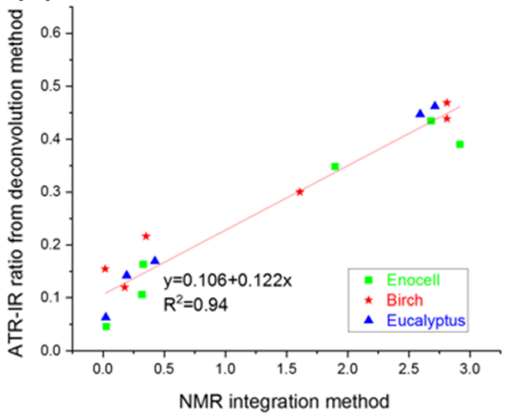

(c)

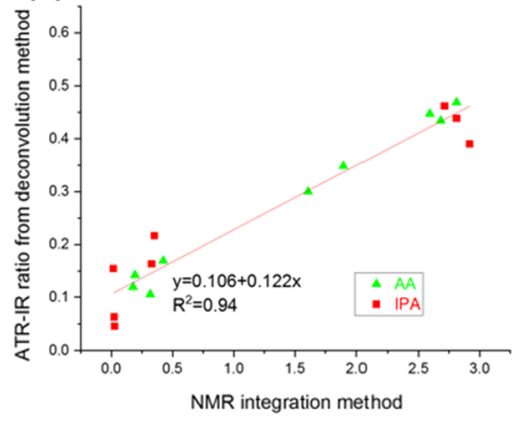

Fig. 4 Linear correlation of DS values by the ${ }^{1} \mathrm{H}$ NMR integration method and ATR-IR ratios by the deconvolution method. Correlation is shown by a the treatment of the pulp, b the pulp source and $\mathbf{c}$ the acetylation reagent. IL-PT-W is the IL pre-treated pulp with a further washing step to remove the IL, before the addition of the acetylating reagent (heterogeneous route) and IL-PT-NW is the non-washed IL pre-treated pulp, which still contains the IL upon addition of the acetylation reagents (homogeneous route) 
of an activating acid catalyst, should give very limited reactivity. It is known that AA is a more reactive reagent than IPA or other vinylic esters (Kakko et al. 2017). AA as acetylating reagent yields higher DS values in comparison to IPA and it is in fact required to get to DS values above 1.5 in the IL-PT-W samples. However, in the case of the IL-PT-NW samples, IPA also allows for conversion to high DS for all pulp samples. Thus, hornification of the pulps after IL removal and drying also seems to have a significant effect on reactivity. Retention of the IL in the swollen matrix could potentially be used to enhance reactivity further. It is also interesting to note that the DS values for the Enocell pulp did not reach the maximum of 3, indicating that the reactions were not truly homogeneous by the end of the reaction. In other words, some crystalline regions retained in the matrix prevent the full reaction, in the absence of a truly homogeneous reaction.

One possible explanation for increased reactivity could also be a reduction in molecular weight. However, the crystallinity removal does not introduce any major decrease in MW during acetylation, as determined by GPC (Fig. 5, Table 1 and Table S5S8). This also agrees with the recent report of Pena et al. (2019). The untreated and pre-treated pulps were all either fully or almost fully soluble in $\mathrm{LiCl} / \mathrm{N}, \mathrm{N}-$ dimethylacetamide (DMA), the gel-permeation chromatography (GPC) mobile phase. This showed only a minor reduction in the number-average molecular weights $\left(\mathrm{DP}_{\mathrm{N}}\right)$ after pre-treatment and washing away the IL (Table 1 and Table S5-S8). The GPC curves show the absence of a higher molecular weight shoulder in the pre-treated kraft pulps. However, this may be due to a residual insolubility of these materials in the mobile phase. Nevertheless, the decrease in $\mathrm{DP}_{\mathrm{N}}$ values are not enough to be especially implicated in the increased reactivity. However, there may of course be some effect.

The removal of crystallinity should also moderate the relative reactivity of hydroxyl units throughout the sample, as certain restrictions caused by the crystallinity are removed during the pre-treatment with $\left[\mathrm{P}_{4444}\right][\mathrm{OAc}]$. To assess this, the regioselectivity of the reaction under heterogeneous conditions was studied by multiplicity-edited HSQC spectroscopic analyses (Fig. 6). Two examples, one low DS (Enocell-Untreated-AA) and one high DS (Enocell-IL-PT-W-AA) were chosen for this study. The low DS sample (DS 0.32 , Fig. 6a) shows characteristic correlations for unmodified cellulose, with no acetylated correlations visible, except close to the baseline noise (Ac- gem- 6 is clearly visible). However, analysis of the diffusionedited ${ }^{1} \mathrm{H}$ spectra plainly show acetylated ${ }^{1} \mathrm{H} 2,{ }^{1} \mathrm{H} 3$ and gem- ${ }^{1} \mathrm{H} 6$ positions on cellulose (see supporting information, Fig. S14)

This is a strong indication of a small amount of cellulose triaceate (CTA) formation, as is expected from acid-catalysed acetylations with AA (Sassi and Chanzy 1995). A small proportion of correlations that corresponding to xylan are quite clear. This is not unexpected as Enocell B-H-PHK-P contains 5\% xylan. The higher DS sample (DS 1.90, Fig. 6b) clearly shows correlations for acetylated hydroxyls. Most of the gem- 6 positions are acetylated. In the 2,3OAc region, most of the acetylated 2 and $3 \mathrm{CH}$ resonance pairs are of the form where $2 \& 3$ hydroxyls are both acetylated at the same time.

Table 2 DS values depending on the acetylating reagent, pulp type and pre-treatment of the pulp

\begin{tabular}{|c|c|c|c|c|c|c|c|c|c|}
\hline \multirow{2}{*}{$\begin{array}{l}\text { Pulp } \\
\text { Treatment }\end{array}$} & \multicolumn{3}{|l|}{ Enocell } & \multicolumn{3}{|l|}{ Birch } & \multicolumn{3}{|l|}{ Eucalyptus } \\
\hline & $\begin{array}{l}\text { Untreated } \\
\text { pulp }\end{array}$ & $\begin{array}{l}\text { IL PT } \\
W^{\mathrm{a}}\end{array}$ & $\begin{array}{l}\text { IL PT } \\
\text { NW }^{\mathrm{b}}\end{array}$ & $\begin{array}{l}\text { Untreated } \\
\text { pulp }\end{array}$ & $\begin{array}{l}\text { IL PT } \\
W^{\mathrm{a}}\end{array}$ & $\begin{array}{l}\text { IL PT } \\
\text { NW }^{\mathrm{b}}\end{array}$ & $\begin{array}{l}\text { Untreated } \\
\text { pulp }\end{array}$ & $\begin{array}{l}\text { IL PT } \\
\mathrm{W}^{\mathrm{a}}\end{array}$ & $\begin{array}{l}\text { IL PT } \\
\text { NW }^{b}\end{array}$ \\
\hline \multicolumn{10}{|c|}{ Acetic anhydride $(A A)$ as acetylating reagent } \\
\hline DS & 0.32 & 1.90 & 2.68 & 0.18 & 1.61 & 2.81 & 0.42 & 0.19 & 2.59 \\
\hline \multicolumn{10}{|c|}{ Isopropenyl acetate (IPA) as acetylating reagent } \\
\hline DS & 0 & 0.33 & 2.92 & 0 & 0.35 & 2.81 & 0 & - & 2.71 \\
\hline
\end{tabular}

${ }^{a}$ IL-PT-W is the IL pre-treated pulp with a further washing step to remove the IL before the addition of the acetylating reagent (heterogeneous route). ${ }^{\mathrm{b}} \mathrm{IL}-\mathrm{PT}-\mathrm{NW}$ is the IL non-washed pre-treated pulp, which still contains the IL upon addition of the acetylation reagents (homogeneous route) 

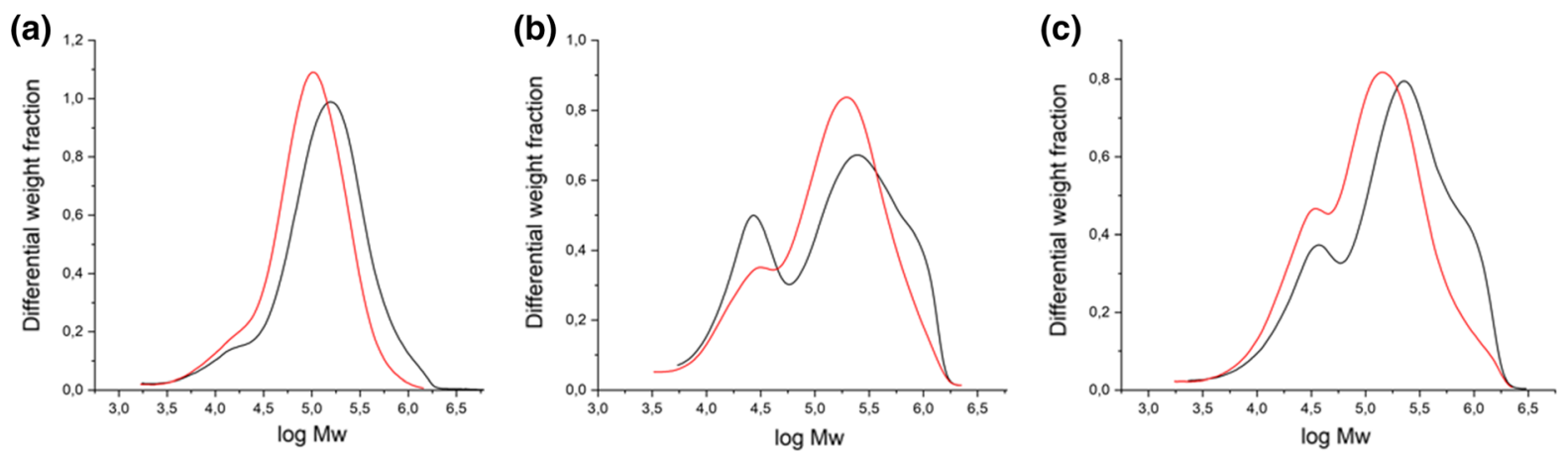

Fig. 5 GPC traces for the untreated (black) and pre-treated (red) pulps: a Enocell, b birch, c eucalyptus

For the Enocell-IL-PT-W-AA sample (high DS), additional resonances are also present which may correspond to alternately unacetylated-acetylated 2 or 3 positions, in cellulose or xylan. Other correlations also remain, corresponding to completely unacetylated cellulose. The visible 4 and $5 \mathrm{CH}$ correlations seem to be split into at least two separate regions each. This splitting is also apparent for the acetylated gem- 6 $\mathrm{CH}$ resonances. By using a combination of cellulose acetate assignments, published by Kono et al. (2015), and 2D HSQC-TOCSY it is possible to trace the separate spin-systems to CTA and cellulose monoacetate (CMA), where only C6-OH is acetylated (see SI Fig. S15). Additional spin-systems were not abundant enough to be identified using this experiment. Thus, the enhancement of reactivity in the heterogeneous system, after IL pre-treatment, seems to offer a little more 'control' over regioselectivity, in particular leading to increased monoacetylation at $\mathrm{C} 6-\mathrm{OH}$. However, the main acetylation product is CTA, consistent with a heterogeneous acetylation. Further mechanistic studies and more detailed NMR analyses are required to better understand this aspect of the reactivity.

\section{AcNH-TEMPO-mediated oxidation}

To assess the effect of $\left[\mathrm{P}_{4444}\right][\mathrm{OAc}]$ pre-treatment for oxidation reactions we employed the method of Kitaoka et al. (1999) (AcNH-TEMPO) as model reaction. The main hypothesis for choosing acidic oxidation conditions was to avoid formation of aldehyde/acetals, which have the potential to halt the reaction at the acetal stage and cross-link the material, preventing complete oxidation and accurate analyses. $\mathrm{NaClO}_{2}$ under mildly acidic conditions is known to

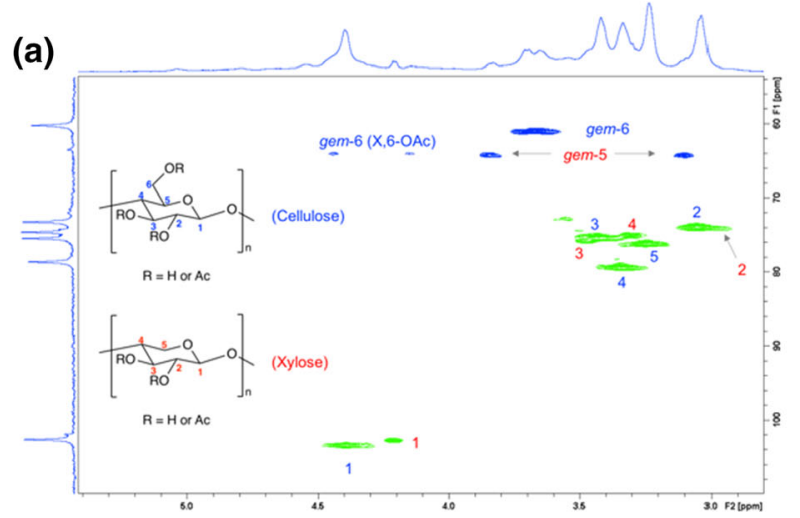

Fig. 6 Multiplicity-edited ${ }^{1} \mathrm{H}_{-}{ }^{13} \mathrm{C}$ HSQC spectra of acetylated Enocell samples: a low DS sample (Enocell-Untreated-AA) in $\left[\mathrm{P}_{4444}\right][\mathrm{OAc}]: D M S O-\mathrm{d}_{6}$ electrolyte (King et al. 2018), and b high DS sample (Enocell-IL-PT-W-AA), in DMSO-d , $_{6}$, at $65{ }^{\circ} \mathrm{C}$. Correlation assignment numbers represent the positions (b)

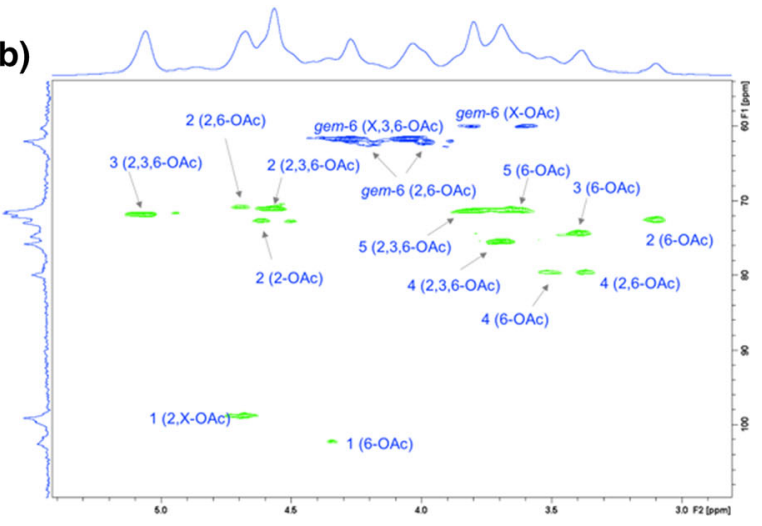

in either cellulose (blue) or xylan (red). The numbers in parenthesis show which hydroxyls in that spin-system are acetylated for that resonance, with ' $\mathrm{X}$ ' representing uncertainty whether additional hydroxyls are acetylated or not. Assignments are aided by, and consistent with, Kono et al. (2015) 
oxidize aldehydes to carboxylic acids and acidic conditions are known to break acetals. Thus, acidic conditions ( $\mathrm{pH}$ 5.8) were chosen, in combination with $\mathrm{NaClO}_{2}$ as co-oxidant, to allow for complete conversion to carboxylate.

For oxidation studies Enocell and birch pulps were chosen as representative examples of different pulp types. After oxidation, water-insoluble fractions with low carboxylate content were separated by centrifugation from the reaction mixture, while water-dispersible fractions with higher carboxylate contents were regenerated from the combined water supernatant by ethanol precipitation and centrifugation (see Sect. 6 of SI). Raw pulps in the oxidation process were not solubilized or did not gel significantly in the reaction media, thus, the amount of regenerated waterdispersible fractions was very low. The freeze-dried samples were analysed by ATR-IR, comparing the peak areas of the carbonyl stretches (Gaussian fitting between 1800 and $1600 \mathrm{~cm}^{-1}$ ) vs the normalized $\mathrm{C}-\mathrm{O}$ backbone stretch (Gaussian fitting between 1200 and $850 \mathrm{~cm}^{-1}$ ). All samples were acidified, under conditions sufficient to protonate the carboxylates $(\mathrm{pH} \mathrm{1})$, prior to the measurements, to minimize peak broadness and changes in absorbance intensity between carboxylic acid and carboxylate forms. In addition, three samples of insoluble fractions, in the $\mathrm{Na}$ salt form, were titrated to determine the carboxylate content, which was then compared against the ATRIR results. A linear correlation was drawn (see SI Fig. S91) to allow for comparison of all samples using IR which was then used for estimation of the remaining carboxylate contents, in mmol/g. (Table 3)

Figure 7 shows that the $\left[\mathrm{P}_{4444}\right][\mathrm{OAc}]$ pre-treatment increased the reactivity of the cellulose samples significantly. Remarkable differences in the carboxylate content between untreated pulps and IL pretreated samples were found. The carboxylate contents for both pulps are clearly decreasing in the following order: IL-PT-NW > IL-PT-W > Untreated pulp.

It is clear that the pre-treatments increase reactivity towards aqueous nitroxyl radical oxidation and that the samples retained in the swollen state, with IL (ILPT-NW), are even much more accessible than the washed samples (IL-PT-W) clearly indicating the benefits of retaining the IL in the cellulose towards oxidation. It is worth noting that results for oxidation of the untreated pulps are a little higher than those reported in literature for a similar oxidation system (Kitaoka et al. 1999; Saito et al. 2010). However, in our case we are using the more active AcNH-TEMPO catalyst, so this is not unexpected.

Table 3 shows the carboxylate contents and mass fraction distributions of the water dispersible and insoluble fractions. The carboxylate contents are also represented by the degrees of oxidation $\left(\%_{\mathrm{DO}}\right)$, the percentage of C6 converted to carboxylate, to better understand how effective the oxidations are. While the $\%_{\text {DO }}$ for the IL pre-treated samples were clearly increased compared to those of the untreated pulps, they are still quite far from the theoretical maximum $\%_{\text {DO }}$ (a carboxylate content of $4.67 \mathrm{mmol} / \mathrm{g}$ for pure cellulose conversion to the sodium polyglucuronate). However, some studies of regenerated cellulose samples (cellulose II) have shown even higher carboxylate contents (Hirota et al. 2009). The carboxylate contents are somewhat lower $(0.87-2.69 \mathrm{mmol} / \mathrm{g})$ in the present study versus $(3.7-4.0 \mathrm{mmol} / \mathrm{g})$ in Hirota et al. (2009).

On the other hand, the morphology of regenerated fibres is much simpler than those of bleached pulp fibres so it is not surprising that the achieved carboxylate contents are higher in the work of Hirota et al. (2009). Clearly the IL pre-treatments allow for a significant increase in reactivity towards nitroxyl radical-type oxidations, based on the amorphous cellulose content. However, retention of the morphology and ultrastructure of the fibres, during the pretreatments, limits the complete reactivity of the systems, compared to the literature regenerated fibres. This is also apparent in the mass recoveries of the insoluble and soluble fractions, with the pre-treated samples showing increased soluble fractions, yet, with an average retention of $60 \%$ insoluble fraction.

Changes in the morphology of the fibre structure were also observed by obtaining microscope images (with cross-polarizing filters) of the oxidised cellulosic material (Na salts), as shown on the Fig. 8. The images of the untreated fibres (Fig. 8a, d) show retention of the fibrous structure after oxidation. This is most likely based on difficulties in extensive swelling of the complex fibre morphology. The IL pre-treatments 
Table $3 \%_{\text {DO }}$ and carboxylate content for different pulp types and their fractional mass distributions, obtained by semi-quantitative ATR-IR analyses, based on peak volume data

\begin{tabular}{|c|c|c|c|c|c|c|c|c|c|c|c|c|}
\hline \multirow{3}{*}{$\begin{array}{l}\text { Pulp } \\
\text { Treatment }\end{array}$} & \multicolumn{6}{|c|}{ Enocell B-H-PHK-P } & \multicolumn{6}{|c|}{ Birch B-H-K-P } \\
\hline & \multicolumn{2}{|c|}{ Untreated pulp } & \multicolumn{2}{|c|}{ IL PT W $W^{\mathrm{a}}$} & \multicolumn{2}{|c|}{ IL PT NW } & \multicolumn{2}{|c|}{ Untreated pulp } & \multicolumn{2}{|c|}{ IL PT Wa } & \multicolumn{2}{|c|}{ IL PT NW } \\
\hline & Insol. $^{\mathrm{e}}$ & Sol. $^{\mathrm{e}}$ & Insol. & Sol. & Insol. & Sol. & Insol. & Sol. & Insol. & Sol. & Insol. & Sol. \\
\hline$\%_{\mathrm{DO}}^{\mathrm{c}}(\%)$ & 22.0 & 34.4 & 18.5 & 37.1 & 47.0 & 63.9 & 23.5 & 36.2 & 38.5 & 61.5 & 36.5 & 62.4 \\
\hline Carboxylate content $(\mathrm{mmol} / \mathrm{g})$ & 1.02 & 1.47 & 0.87 & 1.61 & 2.01 & 2.69 & 1.07 & 1.61 & 1.67 & 2.61 & 1.61 & 2.61 \\
\hline$m^{\mathrm{d}}(\%)$ & 96 & 4 & 40 & 60 & 66 & 34 & 91 & 9 & 76 & 24 & 58 & 42 \\
\hline
\end{tabular}

${ }^{a}$ IL-PT-W is the IL pre-treated pulp with a further washing step to remove the IL before the addition of the acetylating reagent (heterogeneous route). ${ }^{\mathrm{b}} \mathrm{IL}-\mathrm{PT}-\mathrm{NW}$ is the IL non-washed pre-treated pulp, which still contains the IL upon addition of the acetylation reagents (homogeneous route). ${ }^{\mathrm{c}} \%_{\mathrm{DO}}=$ degree of oxidation. ${ }^{\mathrm{d}} m=$ mass of the fraction in percentage. ${ }^{\mathrm{e}}$ Insol. and Sol. are the insoluble and soluble fractions, respectively

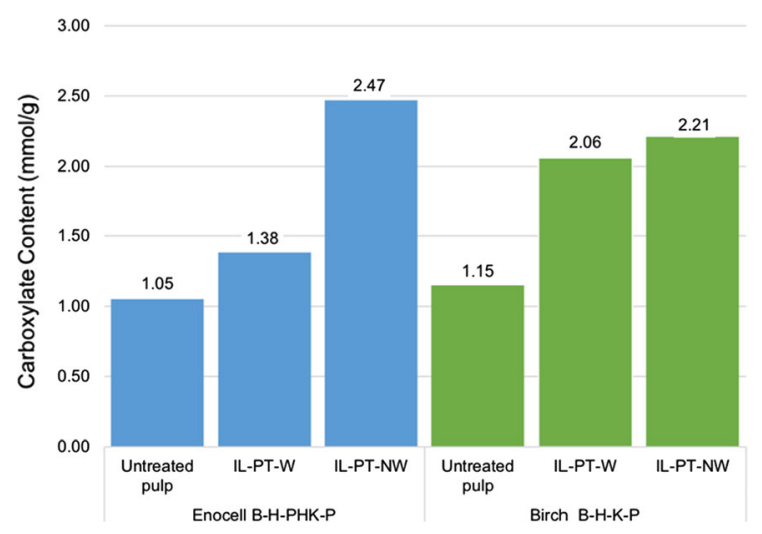

Fig. 7 Total carboxylate content for different pulps and pretreatment types based on ATR-IR peak volume data

(washed (IL-PT-W) and non-washed (IL-PT-NW), Fig. 8b, c, e, f) largely show more thorough swelling of the fibres after oxidation, such that there is significantly reduced residual fibrillar structure. Clearly the IL pre-treatment removes barriers to swelling to a large degree, due to a combination of partial removal of the fibrillar morphology and reduction in crystallinity. Further work needs to be done to decouple these effects, due to the potential broad utility of these oxidations.

\section{IL cost, toxicity, recyclability and cyclical performance}

In any process using complex solvents, cost and toxicity are a major concern. Regarding this specific
IL the cost is dictated by cost of tributylphosphine, which will likely not drop too far below a bulk price of $70 € / \mathrm{kg}$ in the near future. Thus, the IL cost is rather high, which will certainly limit the applicability of this method. However, preliminary toxicity studies have been performed (Ruokonen et al. 2016; Witos et al. 2017) which have identified that the tetrabutylphosphonium homologue does not exhibit any adverse toxicity and is similar to other imidazolium-based structures. In addition, tetraalkylphosphonium-based ionic liquids are much more stable than other cation classes, such as imidazolium or choline-based structures. This is also impacted on the recyclability of the material during process cycling. To initially test this, we cycled the process twice to measure the purity of the IL after each cycle and to assess how the 'decrystallization power' changes. From Fig. 9 it can be observed that the purity of the IL essentially does not change. A small triplet at $0.88 \mathrm{ppm}$ appears, which may indicate some formation of tributylphosphine oxide. However, this does not increase for the 2 nd cycle so may arise from reaction with some basic impurity in the pure IL, which is removed after the first cycle. The CI of the starting pulp is $60.6 \%$. After the first cycle this decreases to $20.3 \%$ (initial results) and $42.6 \%$ (recycling study). After the second cycle the CI is $26.9 \%$. Thus, while there is some variability in the pre-treatment efficiency, significant crystallinity reduction is possible after cycling the IL in the process. 


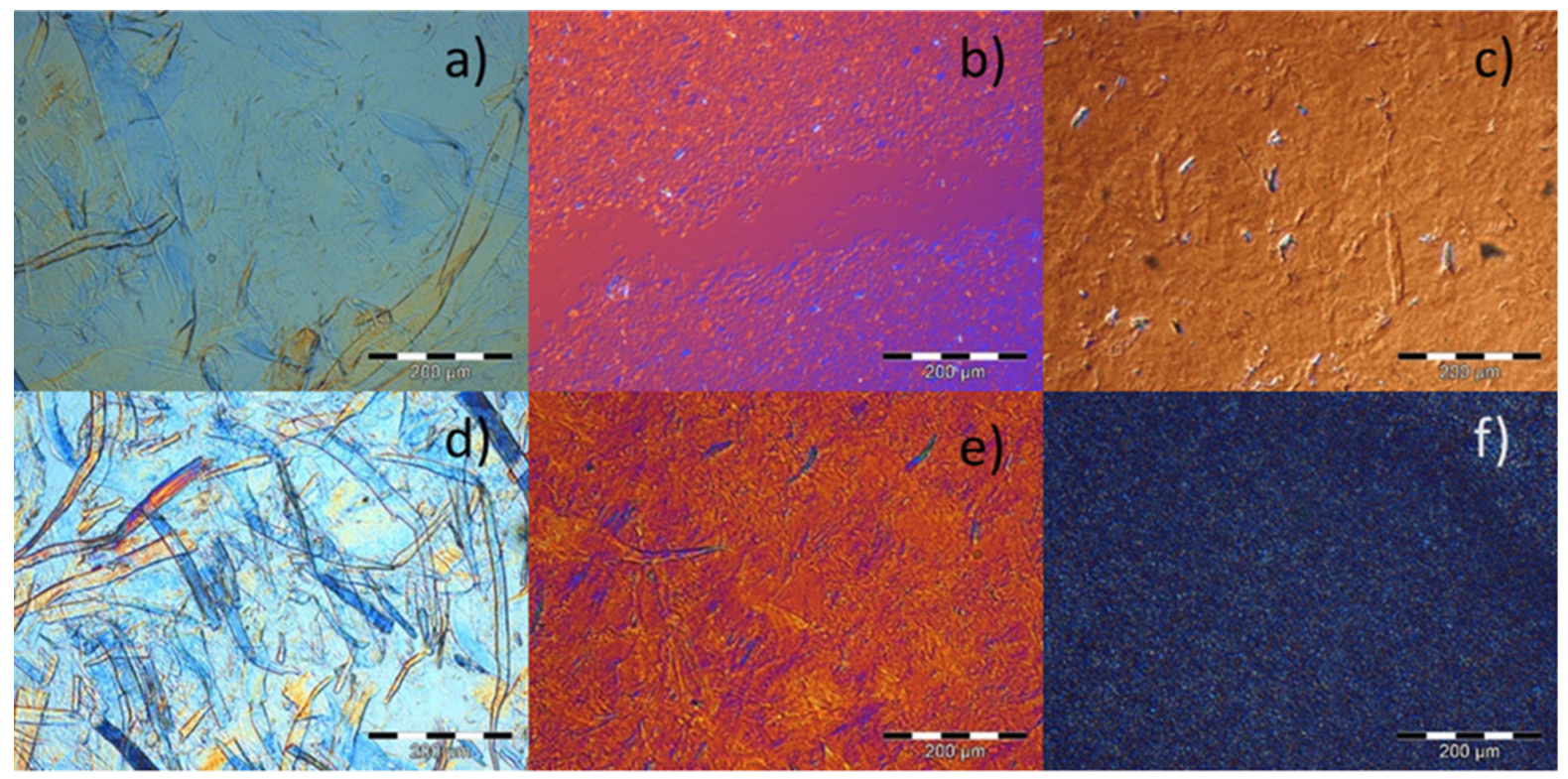

Fig. 8 Microscope images (with variation of the cross-polarising filter angle to improve contrast) of AcNH-TEMPOoxidised cellulosic materials: a untreated Enocell pulp,

\section{Conclusions}

The non-dissolving pre-treatment step with pure $\left[\mathrm{P}_{4444}\right][\mathrm{OAc}]$ reduces efficiently the crystallinity of the kraft pulps to low levels. It was also shown that dissolution and regeneration pre-treatment, with $\left[\mathrm{P}_{4444}\right][\mathrm{OAc}]:$ DMSO (35:65 wt $\left.\%\right)$ is also highly effective for pulp crystallinity and density reduction.

The non-dissolving removal of crystallinity shows an increase in reactivity in non-aqueous (organic swelling) acetylation and aqueous nitroxyl radicalcatalysed oxidation of cellulose, as model reactions. The homogeneous acetylation route, where the ionic liquid was retained in the sample prior to reaction, allowed for almost complete dissolution during the reaction, resulting in high DS values (2.59-2.92) but not full conversion to DS 3 , indicating retention of some crystalline domains. The heterogeneous routes, where the ionic liquid is washed out prior to reaction, yielded mostly insoluble products, except in the case of pre-treated Enocell reacted with acetic anhydride b Enocell-IL-PT-W, c Enocell-IL-PT-NW, d untreated birch pulp, e birch-IL-PT-W, f birch-IL-PT-NW

(DS 1.90) and birch reacted with acetic anhydride (DS 1.61). Isopropenyl acetate was not reactive enough to allow for DS values above 0.35 with any of the heterogeneous reactions. The aqueous acidic AcNHTEMPO-catalysed oxidations yielded carboxylates. These were all heterogeneous reactions. The pretreated samples yielded significantly higher carboxylate contents compared to the untreated pulps. The pretreated samples with the ionic liquid still in the cellulose matrix yielded higher reactivity still, indicating that the presence of the ionic liquid after pretreatment maintains the cellulose in some kind of gelled state, allowing for better penetration of reactants. However, the morphology and ultrastructure of the fibres is still retained somewhat during the pretreatments, limiting the degrees of oxidation on the recovered insoluble fractions. Overall the degrees of oxidation were lower than those achieved for cellulose II fibres (wet-spun from cuprammonium) which clearly have much simpler morphology. 
(a)

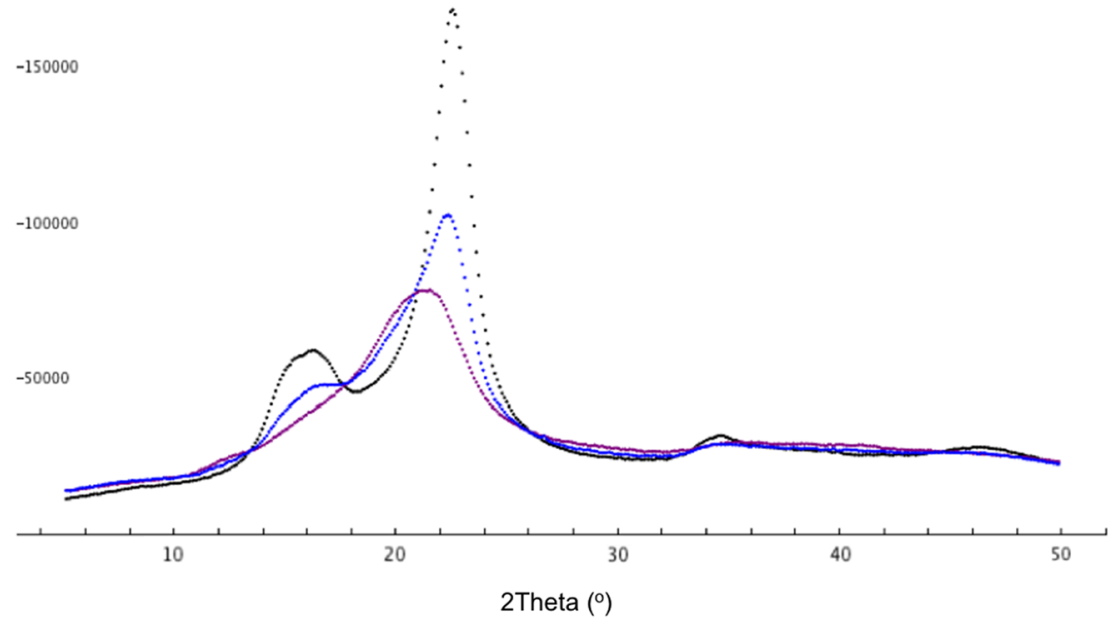

(b)

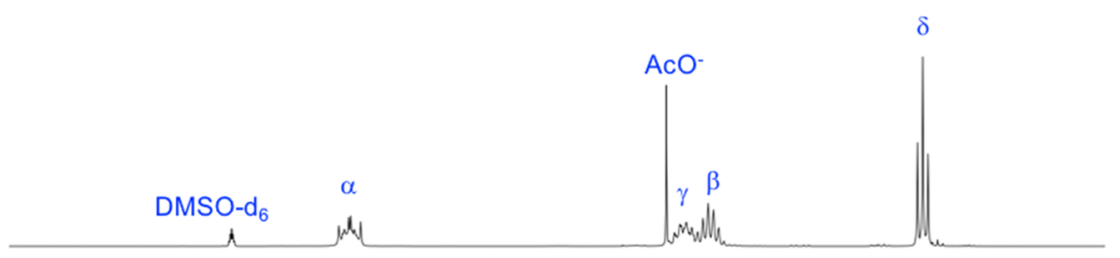

(c)

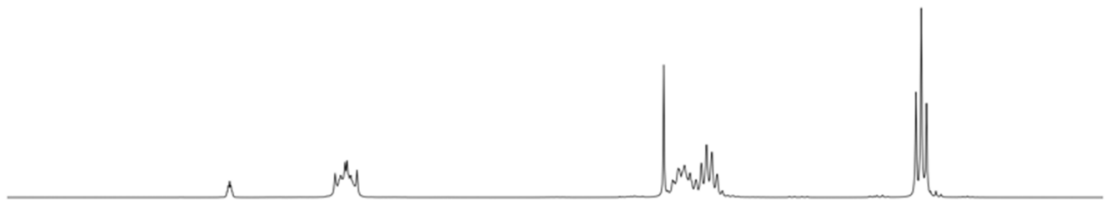

(d)

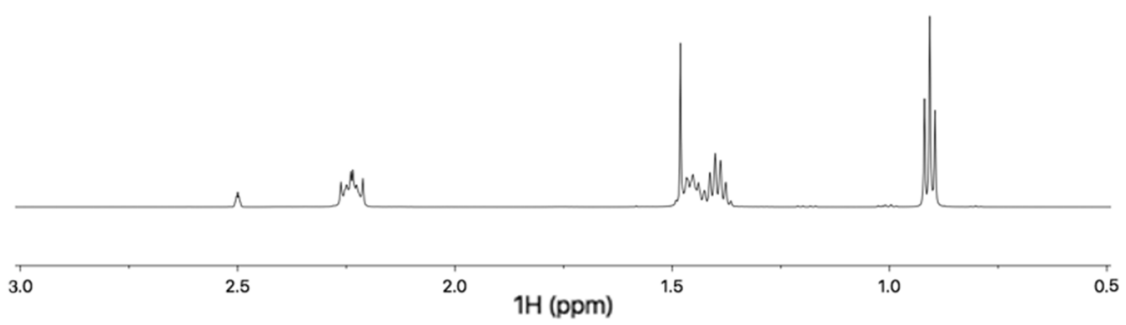

Fig. 9 a WAXS diffractograms showing change in crystallinity from the starting Enocell pulp (black), 1st cycle (blue) and 2nd cycle (purple), including the ${ }^{1} \mathrm{H}$ NMR spectra for $\mathbf{d}$ fresh IL, c 1st cycle and $\mathbf{b} 2$ nd cycle

The applied methodology clearly shows increased reactivity for both non-aqueous and aqueous reactions. It is hoped that this kind of pre-treatment might expand the range of possible heterogeneous reactions applicable to cellulose or to yield more homogenous products, not accessible through the typical heterogeneous reactions applied to high purity celluloses. 
Acknowledgments Open access funding provided by University of Helsinki including Helsinki University Central Hospital. The authors wish to thank UPM-Kymmene and the Finnish Academy, under the projects 'WTF-Click-Nano' (311255) and 'New Forms of Cellulose' (310481), for financial support for this research. The authors also wish to thank Professors French and Nishiyama for advice on the processing of the X-ray data.

\section{Compliance with ethical standards}

Conflict of interest The authors declare that they have no conflict of interest.

Open Access This article is licensed under a Creative Commons Attribution 4.0 International License, which permits use, sharing, adaptation, distribution and reproduction in any medium or format, as long as you give appropriate credit to the original author(s) and the source, provide a link to the Creative Commons licence, and indicate if changes were made. The images or other third party material in this article are included in the article's Creative Commons licence, unless indicated otherwise in a credit line to the material. If material is not included in the article's Creative Commons licence and your intended use is not permitted by statutory regulation or exceeds the permitted use, you will need to obtain permission directly from the copyright holder. To view a copy of this licence, visit http://creativecommons.org/licenses/by/4.0/.

\section{References}

Araki J, Kataoka T, Katsuyama N, Teramoto A, Ito K, Abe K (2006) A preliminary study for fiber spinning of mixed solutions of polyrotaxane and cellulose in a dimethylacetamide/lithium chloride (DMAc/LiCl) solvent system. Polymer 47:8241-8246. https://doi.org/10.1016/j.polymer. 2006.09.060

Chen Yu, Mu T (2019) Application of deep eutectic solvents in biomass pretreatment and conversion. Green Energy Environ 4:95-115. https://doi.org/10.1016/j.gee.2019.01. 012

Chen C-Y, Chen M-J, Zhang X-Q, Liu C-F, Sun R-C (2014) PerO-acetylation of cellulose in dimethyl sulfoxide with catalysed transesterification. J Agric Food Chem 62:3446-3452. https://doi.org/10.1021/jf5002233

Chen J, Xu J, Wang K, Cao X, Sun R (2016) Cellulose acetate fibers prepared from different raw materials with rapid synthesis method. Carbohydr Polym 137:685-692. https:// doi.org/10.1016/j.carbpol.2015.11.034

Chen Y-L, Zhang X, You T-T, Feng X (2019) Deep eutectic solvents (DESs) for cellulose dissolution: a mini-review. Cellulose 26:205-213. https://doi.org/10.1007/s10570018-2130-7

Chowdhury S, Mohan RS, Scott JL (2007) Reactivity of ionic liquids. Tetrahedron 63:2363-2389. https://doi.org/10. $1016 /$ j.tet.2006.11.001
Clough MT, Geyer K, Hunt PA, Son S, Vagt U, Welton T (2015) Ionic liquids: not always innocent solvents for cellulose. Green Chem 17:231-243. https://doi.org/10.1039/ C4GC01955E

De Oliveira Vigier K, Chatel G, Jérôme F (2015) Contribution of deep eutectic solvents for biomass processing: opportunities, challenges, and limitations. ChemCatChem $7: 1250-1260$

Ebner G, Schiehser S, Potthast A, Rosenau T (2008) Side reactions of cellulose with common 1-alkyl-3-methylimidazolium-based ionic liquids. Tetrahedron Lett 49:7322-7324. https://doi.org/10.1016/j.tetlet.2008.10. 052

Fischer S, Leipner H, Thümmler K, Brendler E, Peters J (2003) Inorganic molten salts as solvents for cellulose. Cellulose 10:227-236. https://doi.org/10.1023/A:1025128028462

Fischer S, Thümmler K, Volkert B, Hettrich K, Schmidt I, Fischer K (2008) Properties and applications of cellulose acetate. Macromol Symp 262:89-96. https://doi.org/10. 1002/masy.200850210

French AD (2014) Idealized powder diffraction patterns for cellulose polymorphs. Cellulose 21:885-896. https://doi. org/10.1007/s10570-013-0030-4

Fukaya Y, Sugimoto A, Ohno H (2006) Superior solubility of polysaccharides in low viscosity, polar, and halogen-free 1,3-dialkylimidazolium formates. Biomacromolecules 7:3295-3297. https://doi.org/10.1021/bm060327d

Fukaya Y, Hayashi K, Wada M, Ohno H (2008) Cellulose dissolution with polar ionic liquids under mild conditions: required factors for anions. Green Chem 10:44-46. https:// doi.org/10.1039/B713289A

Fukui S, Ito T, Saito T, Noguchi T, Isogai A (2018) Counterion design of TEMPO-nanocellulose used as filler to improve properties of hydrogenated acrylonitrile-butadiene matrix. Compos Sci Technol 167:339-345. https://doi.org/10. 1016/j.compscitech.2018.08.023

Granström M, Kavakka J, King AWT, Majoinen J, Mäkelä V, Helaja J, Hietala S, Virtanen T, Maunu S-L, Agyropoulos DS, Kilpeläinen I (2008) Tosylation and acylation of cellulose in 1-allyl-3-methylimidazolium chloride. Cellulose 15:481-488. https://doi.org/10.1007/s10570-008-9197-5

Guo X, Liu L, Hu Y, Wu Y (2018) Water vapor sorption properties of TEMPO oxidized and sulfuric acid treated cellulose nanocrystal films. Carbohydr Polym 197:524-530. https://doi.org/10.1016/j.carbpol.2018.06. 027

Heinze T, Liebert TF, Pfeiffer KS, Hussain MA (2003) Unconventional cellulose esters: synthesis, characterization and structure-property relations. Cellulose 10:283-296. https://doi.org/10.1023/A:1025117327970

Hirota M, Tamura N, Saito T, Isogai A (2009) Oxidation of regenerated cellulose with $\mathrm{NaClO}_{2}$ catalyzed by TEMPO and $\mathrm{NaClO}$ under acid-neutral conditions. Carbohydr Polym 78:330-335. https://doi.org/10.1016/j.carbpol. 2009.04.012

Holding AJ, Heikkilä M, Kilpeläinen I, King AWT (2014) Amphiphilic and phase-separable ionic liquids for biomass processing. ChemSusChem 7:1422-1434. https://doi.org/ 10.1002/cssc.201301261

Holding AJ, Mäkelä V, Tolonen L, Sixta H, Kilpeläinen I, King AWT (2016) Solution-state one-and two-dimensional 
NMR spectroscopy of high-molecular-weight cellulose. ChemSusChem 9:880-892. https://doi.org/10.1002/cssc. 201501511

Holding AJ, Parviainen A, Kilpeläinen I, Soto A, King AWT, Rodríguez H (2017) Efficiency of hydrophobic phosphonium ionic liquids and DMSO as recyclable cellulose dissolution and regeneration media. RSC Adv 7:17451-17461. https://doi.org/10.1039/C7RA01662J

Holding AJ, Castro MC, Parviainen A, Rodríguez H, King AWT, Kilpeläinen I (2018) A method for pre-treating cellulosic material. FI Patent, FI20185501

Isogai A, Kato Y (1998) Preparation of polyuronic acid from cellulose by TEMPO-mediated oxidation. Cellulose 5:153-164. https://doi.org/10.1023/A:1009208603673

Isogai T, Saito T, Isogai A (2011) Wood cellulose nanofibrils prepared by TEMPO electro-mediated oxidation. Cellulose 18:421. https://doi.org/10.1007/s10570-010-9484-9

Jebrane M, Sèbe G (2007) A novel simple route to wood acetylation by transesterification with vinyl acetate. Holzforschung 61:143-147. https://doi.org/10.1515/HF.2007. 026

Jebrane M, Pichavant F, Sèbe G (2011) A comparative study on the acetylation of wood by reaction with vinyl acetate and acetic anhydride. Carbohydr Polym 83:339-345. https:// doi.org/10.1016/j.carbpol.2010.07.035

Jelínková M, Briestenský J, Santar I, Říhová B (2002) In vitro and in vivo immunomodulatory effects of microdispersed oxidized cellulose. Int Immunopharmacol 10:1429-1441. https://doi.org/10.1016/S1567-5769(02)00087-5

Jogunola O, Eta V, Hedenström M, Sundman O, Salmi T, Mikkola J-P (2016) Ionic liquid mediated technology for synthesis of cellulose acetates using different co-solvents. Carbohydr Polym 135:341-348. https://doi.org/10.1016/j. carbpol.2015.08.092

Kakko T, King AWT, Kilpeläinen I (2017) Homogeneous esterification of cellulose pulp in [DBNH] [OAc]. Cellulose 24:5341-5354. https://doi.org/10.1007/s10570-017-15215

Kakuchi R, Yamaguchi M, Endo T, Shibata Y, Ninomiya K, Ikai T, Maeda K, Takahashi K (2015) Efficient and rapid direct transesterification reactions of cellulose with isopropenyl acetate in ionic liquids. RSC Adv 5:72071-72074. https:// doi.org/10.1039/C5RA14408F

Kakuchi R, Ito R, Nomura S, Abroshan H, Ninomiya K, Ikai T, Maeda K, Kim HJ, Takahashi K (2017) A mechanistic insight into the organocatalytic properties of imidazoliumbased ionic liquids and a positive co-solvent effect on cellulose modification reactions in an ionic liquid. RSC Adv 7:9423-9430. https://doi.org/10.1039/C6RA28659C

King AWT, Jalomäki J, Granström M, Argyropoulos DS, Heikkinen S, Kilpeläinen I (2010) A new method for rapid degree of substitution and purity determination of chloroform-soluble cellulose esters, using ${ }^{31} \mathrm{P}$ NMR. Anal Methods 2:1499-1505. https://doi.org/10.1039/ C0AY00336K

King AWT, Parviainen A, Karhunen P, Matikainen J, Hauru LKJ, Sixta H, Kilpeläinen I (2012) Relatives and inherent reactivities of imidazolium-based ionic liquids: the implications for lignocellulose processing applications. RSC Adv 2:8020-8026. https://doi.org/10.1039/C2RA21287K
King AWT, Mäkelä V, Kedzior SA, Laaksonen T, Partl GJ, Heikkinen S, Koskela H, Heikkinen HA, Holding AJ, Cranston ED, Kilpeläinen I (2018) Liquid-state NMR analysis of nanocelluloses. Biomacromolecules 19:2708-2720. https://doi.org/10.1021/acs.biomac. 8 b00295

Kitaoka T, Isogai A, Onabe F (1999) Chemical modification of pulp fibers by TEMPO-mediated oxidation. Nord Pulp Pap Res J 4:279-284. https://doi.org/10.3183/npprj-1999-1404-p279-284

Klemm D, Philipp B, Heinze T, Heinze U, Wagenknecht W (1998) Comprehensive cellulose chemistry. Fundamentals and analytical methods. Wiley- $\mathrm{VCH}$, Chichester

Klemm D, Heublein B, Fink HP, Bohn A (2005) Cellulose: fascinating biopolymer and sustainable raw material. Angew Chem Int Ed 44:3358-3393. https://doi.org/10. 1002/anie. 200460587

Kono H, Hashimoto H, Shimizu Y (2015) NMR characterization of cellulose acetate: chemical shift assignments, substituent effects, and chemical shift additivity. Carbohydr Polym 118:91-100. https://doi.org/10.1016/j.carbpol. 2014.11.004

Krässig HA (1993) Cellulose: structure, accessibility and reactivity. Gordon and Breach Science Publishers, New York

Lasse K, Parviainen A, Deb S, Lawoko M, Gorlov M, Kilpeläinen I, King AWT (2013) On the solubility of wood in non-derivatising ionic liquids. Green Chem 15:2374-2378. https://doi.org/10.1039/C3GC41273C

Li J, Zhang L-P, Peng F, Bian J, Yuan TQ, Xu F, Sun R-C (2009) Microwave-assisted solvent-free acetylation of cellulose with acetic anhydride in the presence of iodine as a catalyst. Molecules 14:3551-3566. https://doi.org/10.3390/ molecules 14093551

Li P, Sirviö JA, Haapala A, Liimatainen H (2017) Cellulose nanofibrils from nonderivatising urea-based deep eutectic solvent pretreatments. Appl Mater Interfaces 9:2846-2855

Li P, Sirviö JA, Asante B, Liimatainen H (2018) Recyclable deep eutectic solvent for the production of cationic nanocelluloses. Carbohydr Polym 199:219-227

Nam S, French AD, Condon BD, Concha M (2016) Segal crystallinity index revised by the simulation of X-ray diffraction patterns of cotton cellulose I $\beta$ and cellulose II. Carbohydr Polym 135:1-9. https://doi.org/10.1016/j. carbpol.2015.08.035

Nawaz H, Pires PAR, Bioni TA, Arêas EPG, El-Seoud OA (2014) Mixed solvents for cellulose derivatization under homogeneous conditions: kinetic, spectroscopic, and theoretical studios on the acetylation of the biopolymer in binary mixtures of an ionic liquid and molecular solvents. Cellulose 21:1193-1204. https://doi.org/10.1007/s10570014-0184-8

Orelma H, Filpponen I, Johansson L-S, Österberg M, Rojas OJ, Laine J (2012) Surface functionalized nanofibrillar cellulose (NFC) film as a platform for immunoassays and diagnostics. Biointerphases 7:61-73. https://doi.org/10. 1007/s13758-012-0061-7

Pan Y, Birdsey RA, Fang J, Houghton R, Kauppi PE, Kurz WA, Phillips OL, Shvidenko A, Lewis SL, Canadell JG, Ciais P, Jackson RB, Pacala SW, McGuire AD, Pia S, Rautiainen A, Sitch S, Hayes D (2011) A large and persistent carbon 
sink in the world's forest. Science 333:988-993. https:// doi.org/10.1126/science.1201609

Park S, Baker JO, Himmel ME, Parilla PA, Johnson DK (2010) Cellulose crystallinity index: measurement techniques and their impact on interpreting cellulose performance. Biotehnol Biofuels 3:10-20. https://doi.org/10.1186/17546834-3-10

Parviainen A, King AWT, Mutikainen I, Hummel M, Selg C, Hauru LKJ, Sixta H, Kilpeläinen I (2013) Predicting cellulose solvating capabilities of acid-base conjugate ionic liquids. ChemSusChem 6:2161-2169. https://doi.org/10. 1002/cssc. 201300143

Pena CA, Soto A, King AWT, Rodríguez H (2019) Improved reactivity o cellulose via its crystallinity reduction by nondissolving pretreatment with an ionic liquid. ACS Sustain Chem Eng 7:9164-9171. https://doi.org/10.1021/ acssuschemeng.8b06357

Philipp B, Nehls I, Wagenknecht W (1987) Carbon-13 NMR spectroscopic study of the homogeneous sulfation of cellulose and xylan in the dinitrogen tetroxide-DMF system. Carbohydr Res 164:107-116. https://doi.org/10.1016/ 0008-6215(87)80123-4

Ramos LA, Frollini E, Heinze T (2005) Carboxymethylation of cellulose in the new solvent dimethyl sulfoxide/tetrabutylammoium fluoride. Carbohydr Polym 60:259-267. https:// doi.org/10.1016/j.carbpol.2005.01.010

Rico del Cerro D, Mera-Adasme R, King AWT, Perea-Buceta JE, Heikkinen S, Hase T, Sundholm D, Wähälä K (2018) On the mechanism of the reactivity of 1,3-dialkylimidazolium salts under basic to acidic conditions: a combined kinetic and computational study. Angew Chem Int Ed 57:11613-11617. https://doi.org/10.1002/anie.201805016

Rosenau T, Potthast A, Sixta H, Kosma P (2001) The chemistry of side reactions and byproduct formation in the system NMMO/cellulose (Lyocell process). Prog Polym Sci 9:1763-1837. https://doi.org/10.1016/S00796700(01)00023-5

Ruokonen S-K, Sanwald C, Sundvik M, Polnick S, Vyavaharkar $\mathrm{K}$, Duša F, Holding AJ, King AWT, Kilpeläinen I, Lämmerhofer M, Panula P, Wiedmer SK (2016) Effect of ionic liquids on zebrafish (Danio rerio) viability, behaviour, and histology; correlation between toxicity and ionic liquid aggregation. Environ Sci Technol 50:7116-7125. https://doi.org/10.1021/acs.est.5b06107

Saito T, Hirota M, Tamura N, Isogai A (2010) Oxidation of bleached wood pulp by TEMPO/ $\mathrm{NaClO} / \mathrm{NaClO}_{2}$ system: effect of the oxidation conditions on carboxylate content and degree of polymerization. J Wood Sci 56:227-232. https://doi.org/10.1007/s10086-009-1092-7

Sassi J-F, Chanzy H (1995) Ultrastructural aspects of the acetylation of cellulose. Cellulose 2:111-127. https://doi. org/10.1007/BF00816384

Segal L, Creely JJ, Martin AE Jr, Conrad CM (1959) An empirical method for estimating the degree of crystallinity of native cellulose using the X-ray diffractometer. Text Res J 29:786-794. https://doi.org/10.1177/ 004051755902901003

Siódmiak T, Marszall MP, Proszowska A (2012) Ionic liquids. A new strategy in pharmaceutical synthesis. Mini-Rev Org Chem 9:203-208. https://doi.org/10.2174/ 157019312800604698

Skoda-Földes R (2014) The use of supported acidic ionic liquids in organic synthesis. Molecules 19:8840-8884. https://doi. org/10.3390/molecules 19078840

Swatloski PR, Spear SK, Holbrey JD, Rogers RD (2002) Dissolution of cellulose with ionic liquids. J Am Chem Soc 124:4974-4975. https://doi.org/10.1021/ja025790m

Witos J, Russo G, Ruokonen S-K, Wiedmer SK (2017) Unraveling interactions between ionic liquids and phospholipid vesicles using nanoplasmic sensing. Langmuir 33:1066-1076. https://doi.org/10.1021/acs.langmuir. $6 \mathrm{~b} 04359$

Wojdyr M (2010) Fityk: a general-purpose peak fitting program. J Appl Crystallogr 43:1126-1128. https://doi.org/10.1107/ S0021889810030499

Wu J, Zhang J, Zhang H, He J, Ren Q, Guo M (2004) Homogeneous acetylation of cellulose in a new ionic liquid. Biomacromolecules 5:266-268. https://doi.org/10.1021/ bm034398d

Zhu S, Wu Y, Chen Q, Yu Z, Wang C, Jin S, Ding Y, Wu G (2006) Dissolution of cellulose with ionic liquids and its application: a mini-review. Green Chem 8:325-327. https://doi.org/10.1039/B601395C

Zimnitsky DS, Yurkshtovich TL, Bychkovsky PM (2006) Adsorption of zwitterion drugs onto oxidized cellulose. J Colloid Interface Sci 1:33-40. https://doi.org/10.1016/j. jcis.2005.07.058

Publisher's Note Springer Nature remains neutral with regard to jurisdictional claims in published maps and institutional affiliations. 